\title{
Calcium Magneto-Optical Trap Loaded from a Decelerated Atomic Beam
}

\author{
Reinaldo L. Cavasso Filho, Daniela A. Manoel, Davi R. Ortega, \\ Artemio Scalabrin, Daniel Pereira, and Flavio C. Cruz \\ Instituto de Física 'Gleb Wataghin', Universidade Estadual de Campinas, \\ CP.6165, Campinas, SP, 13083-970, Brazil
}

Received on 10 October, 2002.

\begin{abstract}
We describe a new system for laser cooling and trapping of neutral Calcium atoms employing the ${ }^{1} S_{0}-{ }^{1} P_{1}$ resonant transition at $423 \mathrm{~nm}$. An on-axis magneto-optical trap (MOT) is loaded from a Zeeman decelerated atomic beam. When a single laser is used, in order to avoid perturbation of the trap by the deceleration laser beam, this one has been tightly focused near the MOT center, with a waist size much smaller than the atomic cloud. In order to test the efficiency of this novel technique, we have then employed a second, independent decelerating laser, with a profile mode matched to the atomic beam. For an oven temperature of $580^{\circ} \mathrm{C}$ this system can load 1.2 (2) x $10^{7}$ atoms in 16 (1) $\mathrm{ms}$. By the spatial extension of the atomic cloud the one dimension rms velocity was estimated to be $136(12) \mathrm{cm} / \mathrm{s}$, corresponding to a temperature of 9 (2) $\mathrm{mK}$. The variation of the number of trapped atoms as a function of laser detuning and intensity, trap magnetic field gradient and oven temperature is analyzed. Spatial structures of the trapped atoms, like stable rings created by vortex forces, have been observed. This is the first time that these structures, already observed in alkali-metal elements, are reported in MOTs of alkaline-earth elements.
\end{abstract}

\section{Introduction}

Laser cooling and trapping of alkaline-earth atoms is receiving increasing attention in the past years due, for example, to interests in optical frequency standards and clocks, investigation of cold collisions or the possibility to extend the quantum degenerate regime to these elements, possibly by all-optical means. The almost closed ${ }^{1} S_{0}-{ }^{1} P_{1}$ resonant transition, between the ground state and the first singlet state of these elements has a large natural width $(34.6 \mathrm{MHz}$ for $\mathrm{Ca}$ ), which makes it excellent for laser manipulation by radiation pressure. However, the wavelength of this transition requires a laser in the blue or violet region (423 $\mathrm{nm}$ for $\mathrm{Ca}$ ), which also in principle needs to be powerful because saturation parameters of atomic transitions are correspondingly higher in this region. This partially accounts for the relatively small number of groups that had or have been working in laser cooling and trapping of alkaline-earths and, in particular, Calcium [1-4]. On the other hand, the spin forbidden ${ }^{1} S_{0}-{ }^{3} P_{1}$ intercombination transition of alkalineearths, also departing from the ground state, has low transition rate and consequent narrow linewidth. For Calcium, this linewidth is only $408 \mathrm{~Hz}$ [1]. This transition has been used for experiments in high resolution and precision spectroscopy [5], atomic interferometry [6], optical frequency standards and clocks [3, 7], and laser cooling and trapping into microKelvin temperatures [8, 9]. The recent advent of frequency measurements in the optical region with femtosecond lasers [10] has made Calcium a promising candidate for a unified standard of length, frequency and time.
In this paper we give a detailed characterization of our system for laser cooling and trapping Calcium atoms with the ${ }^{1} S_{0}-{ }^{1} P_{1}$ transition at $423 \mathrm{~nm}$. Among several possible applications, we intend to use this system for an optical clock based on the intercombination transition at 456 THz. In Section 2, we describe the experimental apparatus for production of the atomic beam, the Zeeman slower and the on-axis MOT. In Section 3, we discuss how we estimate the number of trapped atoms when the atomic cloud is comparable to the laser waist sizes. An analysis of the number of trapped atoms with the slower laser power, detuning and magnetic field gradient, when decelerating and trapping with the same laser, is presented in Section 4. We show that $10 \mathrm{~mW}$ at $423 \mathrm{~nm}$ is enough power to decelerate and trap Calcium. Spatial structures of trapped atoms, created by vortex forces, are also reported in this section. In Section 5, we discuss our results for independent atomic beam deceleration with a second laser. The temperature of the atomic ensemble is estimated by the Gaussian profile of the atomic cloud in Section 6. Finally, the conclusions are summarized in Section 7.

\section{Experimental Apparatus}

Figure 1 shows a schematic diagram of the experimental apparatus. The atomic beam is produced in a stainless steel cylindrical oven with an exit aperture of $2 \mathrm{~mm}$ [11]. The oven chamber is connected to a $240 \mathrm{l} / \mathrm{s}$ turbo pump and has two sapphire optical windows which allows saturation ab- 
sorption spectroscopy in the high atomic flux environment near the oven. The atomic beam collimation is defined by another $2 \mathrm{~mm}$ aperture, $15 \mathrm{~cm}$ apart from the oven one. It connects the oven chamber with an all-glass chamber for the atomic beam and MOT. Screws are used to fix the oven chamber to the table and to adjust the beam angle. Most of the atoms are lost by not passing through this collimating hole. Although the generation of the atomic beam in this way is not very efficient, the system can be operated continuously at temperatures near $480^{\circ} \mathrm{C}$ by several months without need for filling Calcium [12]. At these temperatures, the flux of atoms in the atomic beam was estimated to be 3.2(5) $\mathrm{x} 10^{12} s^{-1}$. By operating the system at temperatures higher than $600^{\circ} \mathrm{C}$, the useful time drops to around one week, under daily use.

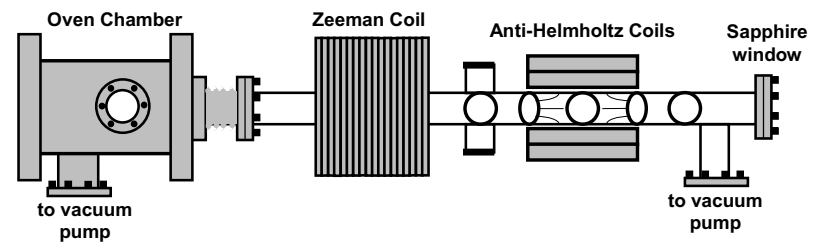

Figure 1. Schematic diagram of the atomic beam and MOT experimental apparatus. It shows, from left to right: the oven chamber, the slower and MOT glass chamber with 14 AR coated windows and one Sapphire window at the right end. Also shown is the 18 sections Zeeman magnet and the anti-Helmholtz trap coils.

The atomic beam slower and MOT chamber consist of a $90 \mathrm{~cm}$ long PIREX glass tube with 14 optical windows, AR coated for $423 \mathrm{~nm}$ and $657 \mathrm{~nm}$, plus a Sapphire window at the tube end, for coupling the decelerating laser beam. The $22 \mathrm{~cm}$ long Zeeman magnet is composed of 18 independent sections, $1 \mathrm{~cm}$ long each, rolled in a water-cooled copper cylindrical basis. With all section at $1 \mathrm{~A}$, for example, the maximum magnetic field is 780 Gauss. This allows deceleration of all atoms with velocities lower than $460 \mathrm{~m} / \mathrm{s}$, which corresponds to $13 \%$ of the velocity distribution when the oven is at $480^{\circ} \mathrm{C}$. By adjusting the currents of each section it is possible to optimize the magnetic field profile for deceleration. After the Zeeman magnet, $47 \mathrm{~cm}$ downstream from the oven chamber collimation aperture, there are two pairs of AR coated windows. They can be used for a 2-D optical molasses to transversally cool the atomic beam, a 2-D trap to also compress it, or even for spectroscopy. After these windows, we have the MOT chamber, $60 \mathrm{~cm}$ downstream from the collimation aperture, with six AR coated windows in the horizontal plane and two in the vertical plane. One horizontal pair of windows, perpendicular to the atomic beam direction, is used for spectroscopy or for monitoring the fluorescence with a calibrated photodiode and a CCD camera. The other windows are used for the trapping laser beams. Around the vertical windows, two air-cooled anti-Helmholtz coils are attached in the MOT chamber. For a current of 1 A, these coils produce vertical and horizontal field gradients of 63 and 32 Gauss $/ \mathrm{cm}$. After $13 \mathrm{~cm}$ from the MOT center we have an additional horizontal pair of AR coated windows, which can also be used for spectroscopy. After this, there is a connection to another $240 \mathrm{l} / \mathrm{s}$ turbo pump. At the tube end, $90 \mathrm{~cm}$ from the oven chamber aperture, we have a sapphire window. This window is heated up to $250^{\circ} \mathrm{C}$ to prevent deposition of Calcium atoms from the atomic beam [13]. Typical background pressure in the MOT chamber is $5 \times 10^{-8}$ mbar, when the oven is switched off and $10^{-7}$ mbar when the atomic beam is on.

To generate the coherent radiation at $423 \mathrm{~nm}$, for deceleration, cooling and trapping, we are using a homemade frequency doubled and stabilized Ti:Sapphire laser [14]. With this laser, we are able to generate $65 \mathrm{~mW}$ of useful power at $423 \mathrm{~nm}$, when pumping with 5 Watts at $532 \mathrm{~nm}$. The thermal drift of the Ti:Sapphire laser locked to a Fabry Perot quartz cavity is less than $30 \mathrm{MHz}$ per hour in normal operating conditions, without any temperature stabilization or environmental isolation. Therefore this laser does not require further stabilization to an atomic reference line. The 2 nd harmonic beam is split in four beams of same power. One of these is used for Zeeman deceleration and the other three are used in the standard retroreflected $\sigma_{+}-\sigma_{-}$3D MOT configuration [15]. The power unbalance in each direction due to window losses is less than $5 \%$, which has no significant effect in the position of the atomic cloud. The laser intensity has a Gaussian shape:

$$
I(r)=I_{0} \exp \left(-r^{2} / w^{2}\right)
$$

where $w$ is the waist size and $r$ is the transverse direction. The six trap beams have equal waist sizes, measured to be $1.40(5) \mathrm{mm}$. Peak intensities $I_{0}$ up to $200 \mathrm{~mW} / \mathrm{cm}^{2}$ per each MOT beam can be obtained.

We have implemented an on-axis trap, where the MOT is located within the atomic beam, which has a diameter of $2 \mathrm{~cm}$ at the MOT position. Several configurations have been tested with similar results, with the MOT more or less centered with respect to the atomic beam. One of the problems of an on-axis trap, loaded from an atomic beam decelerated with the same laser, is that this one very much disturbs the trapped atomic cloud. One solution to this problem is to shift the slowing laser frequency far from resonance, for example with an acousto-optical modulator. An adjustment of the Zeeman slower magnetic field is then required to bring the outcome velocity of the decelerated atoms to the same value [16]. Another solution uses a slower laser with a central "dark spot" [17]. Both share the problem of laser power consumption, which is not desired when dealing with alkaline-earth elements. We have employed another solution, which consists in focusing the counterpropagating slower laser beam to a spot size of about $20 \mu \mathrm{m}$, dislocated $2 \mathrm{~mm}$ from the trap in the transverse plane. This avoids any perturbation of the trapped atoms due to radiation pressure of the decelerating laser. The slowed atoms are captured in a traditional $\sigma_{+}-\sigma_{-}$3D MOT configuration [15].

\section{Number of Trapped Atoms}

The number of trapped atoms is estimated by the scattered light power. In the presence of six laser beams of same intensity $I$, the scattered power by one atom is given simply by the product of the photon energy and the scattering rate 
[18]:

$$
P_{1}=h \nu \frac{\gamma}{2}\left[\frac{6 I / I_{s}}{1+6 I / I_{s}+4 \delta^{2} / \gamma^{2}}\right],
$$

where $\mathrm{h}$ is the Planck constant, $\nu$ is the photon frequency, $\gamma$ is the angular natural linewidth, $I_{s}$ is the saturation intensity, and $\delta$ is the laser detuning. For the Calcium resonant transition ${ }^{1} S_{0}-{ }^{1} P_{1}, \nu=710 \mathrm{THz}, \gamma=234.6 \mathrm{MHz}$, and $I_{s}=59.9$ $\mathrm{mW} / \mathrm{cm} 2$ [19]. Assuming that all atoms are subjected to the same total laser intensity $6 I$, then the total scattered power is simply $N P_{1}$. This holds only when the laser waist size is large in comparison with the atomic cloud extension, in which case $I=I_{0}$. In a more realistic situation, the atoms spatial extent is not negligible with respect to the laser waist sizes and we have to take into account the Gaussian laser profile over the atomic density distribution $n(r)$. In other words, we have to consider an effective intensity as an ensemble average:

$$
I_{e f f}=\frac{1}{N} \int I(r) n(r) d^{3} r .
$$

In the case of alkaline-earth elements, for densities below $10^{11} \mathrm{~cm}^{-3}$, the spatial distribution of the atomic cloud is Gaussian [20]. The MOT "spring constant" depends directly on the magnetic field gradient [19]. Therefore the cloud is smaller in the direction of the higher magnetic field gradient, $z$, and larger in the plane where the gradient is lower, plane $x y$. For moderated densities we can thus write:

$$
n(r)=n_{0} \exp \left(\frac{-z^{2}}{a_{z}}\right) \exp \left[-\left(x^{2}+y^{2}\right) / a^{2}\right],
$$

where $n_{0}$ is the peak density, $a_{z}$ is the width of the atomic cloud in the vertical direction and $a$ is the width in the horizontal plane. In our case, we typically have a vertical field gradient of $63 \mathrm{Gauss} / \mathrm{cm}$ and half of this value in the horizontal plane. Integration of Eq.(4) in an infinite volume give us the total number of atoms as $N=n_{0} \pi^{3 / 2} a_{z} a^{2}$. Assuming that all six laser beams have the same intensity and waist, we get from Eq.(3) the following effective total intensity:

$$
I_{e f f}=\frac{2 I_{0}}{\sqrt{1+a^{2} / w^{2}}}\left(\frac{1}{\sqrt{1+a^{2} / w^{2}}}+\frac{2}{\sqrt{1+a_{z}^{2} / w^{2}}}\right) \text {, }
$$

which for small atomic clouds goes to $6 I_{0}$, as expected. The ensemble average power scattered per atom is then given by Eq.(2) by just replacing the term $6 I$ by the effective intensity, $I_{\text {eff }}$. We have estimated in this way the number of atoms as a function of several parameters.

\section{Atomic Beam Deceleration With a Focused Laser}

To measure the number and the spatial extension of the trapped atoms we use the two horizontal windows of the MOT chamber with the CCD camera and the calibrated photodiode. Figure 2 shows a series of ten pictures of the trapping region for several laser detunings. For the first picture in the left the laser detuning is $84 \mathrm{MHz}$ to the blue side of the resonance, changing by $28 \mathrm{MHz}$ to the red for each picture in the sequence. In these pictures we also observe the Doppler-free atomic beam fluorescence due to the vertical laser beams. In the first three pictures, with decreasing blue detunings, we observe two well defined clouds separated spatially. The reason for this effect is the Zeeman splitting of the excited ${ }^{1} P_{1}$ level in the trap quadrupole magnetic field and the symmetric laser configuration $\sigma_{+}-\sigma_{-}$in this direction. At resonance, the fluorescence of the $\sigma_{+}$beam occurs at same position of the $\sigma_{-}$beam. With this spatial splitting we estimate the atomic resonance with an uncertainty of 5 $\mathrm{MHz}$, for a field gradient of $63 \mathrm{Gauss} / \mathrm{cm}$. This accuracy is limited by the divergence of the atomic beam, estimated to be $5 \mathrm{mrad}$. The resolution of the CCD camera, near $40 \mu \mathrm{m}$ in our case, plays a minor role in the uncertainty. In this way, we are determining the atomic resonance just by looking at this Zeeman spatial splitting in the CCD image. The detuning is then given by the excursion of the PZT that tunes the reference Fabry-Perot cavity of the Ti:sapphire laser, which has a coefficient of $27.8 \mathrm{MHz} /$ Volt at the $2^{\text {nd }}$ harmonic.

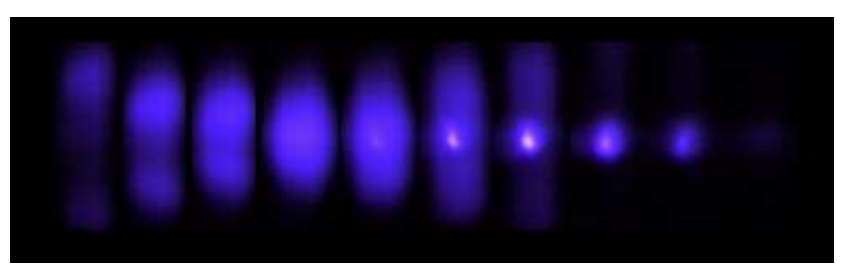

Figure 2. Pictures of the Calcium MOT central region taken under ordinary snapshot conditions for several laser detunings. The first picture in the left corresponds to a blue detuning of $84 \mathrm{MHz}$. In the next ones the detuning changes by $28 \mathrm{MHz}$ to the red from picture to picture.

By analyzing the central lines of the CCD image, we obtain the horizontal and vertical spatial distributions, as showed in Figure 3, for a red detuning of $45 \mathrm{MHz}$ and a field gradient of $41 \mathrm{Gauss} / \mathrm{cm}$. To obtain these intensity profiles we subtracted from the picture of the trapped atoms another picture in the same conditions, but without the slower laser beam, to get ride of the atomic beam background fluorescence.

Figures 2 and 3, showing near spherical Gaussian distributions, were obtained with a careful alignment of the trapping laser beams. For small misalignments, we obtain different spatial structures of the trapped atoms. Figure 4 shows a situation of alignment that produces a stable ring of trapped atoms. These kinds of structures have already been reported for MOTs of alkali-metal elements [21]. The explanation of these spatial modes is related to a vortex force that appears with the misalignment of the MOT beams [21, 22]. In our situation, where the laser intensity is high and the waist size is small, such structures are easily observed even for small misalignments. The theoretical models that explain this behavior assume a two level atom, with J" $=0$ and $\mathrm{J}$ '=1 levels, which is far from the real case for alkali-metal elements already experimentally studied. On the other hand, an analysis of such structures in MOTs of alkaline-earth elements can provide us a direct comparison with the models. 


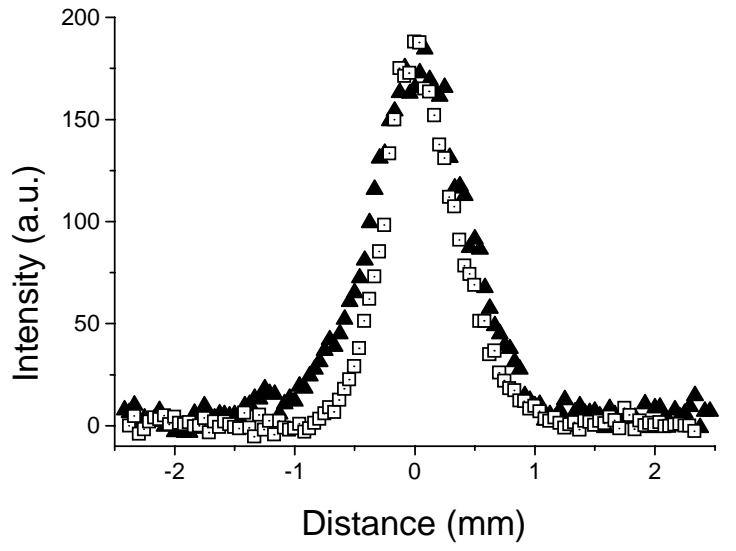

Figure 3. Atomic cloud vertical (triangles) and horizontal (squares) distribution.

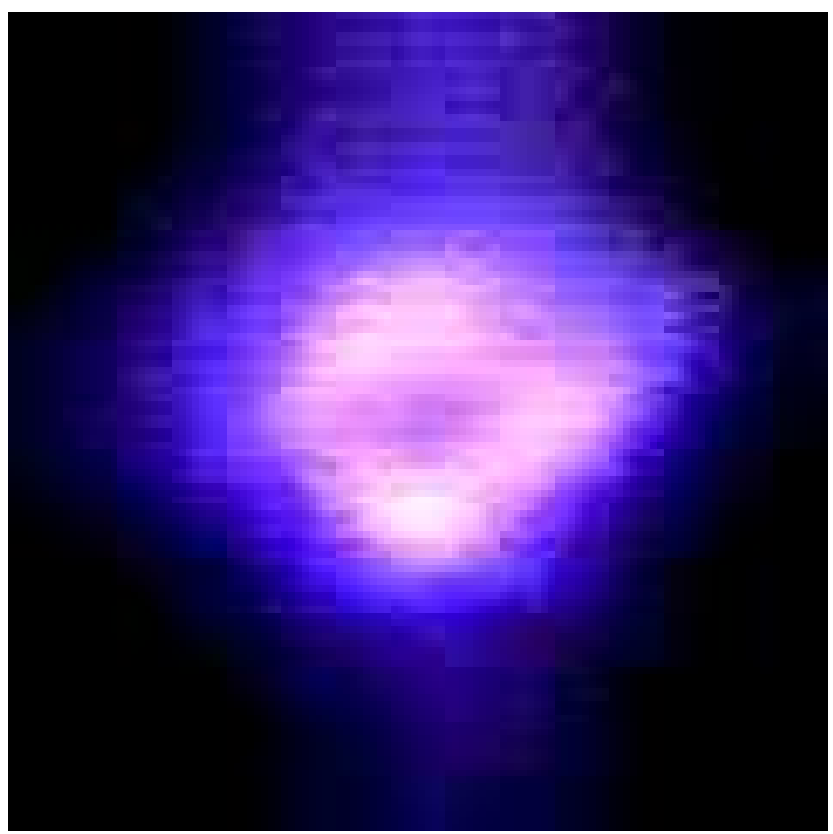

Figure 4. Picture of the MOT central region $\left(5 \times 5 \mathrm{~mm}^{2}\right)$ showing a stable ring structure of trapped atoms.

To measure the total number of trapped atoms, we image the atomic cloud in a calibrated photodiode and take into account the considerations discussed in section 3 . To get ride of the background signal due to the atomic beam fluorescence we modulate the slower laser beam with a mechanical shutter. By positioning this shutter at a telescope focus, we obtain a $100 \mu$ s switch-on or off time. The laser remains on or off for around $100 \mathrm{~ms}$.

In Figure 5 we present the number of trapped atoms as a function of laser detuning, for $10 \mathrm{~mW}$ per each trap and slower beam, a vertical field gradient of $63 \mathrm{Gauss} / \mathrm{cm}$ and oven temperature of $480{ }^{\circ} \mathrm{C}$. The peak number of 9 (1) $\times 10^{5}$ atoms occurs for a red detuning of 97 (5) $\mathrm{MHz}$, or 2.8 (1) atomic linewidths. The uncertainty in the estimated number comes mainly from the uncertainty in the solid angle covered by the detection system. For a fixed total laser power, the optimum detuning that maximizes the number does not change significantly with the field gradient, although the maximum atom number does, as we can see in Figure 6.

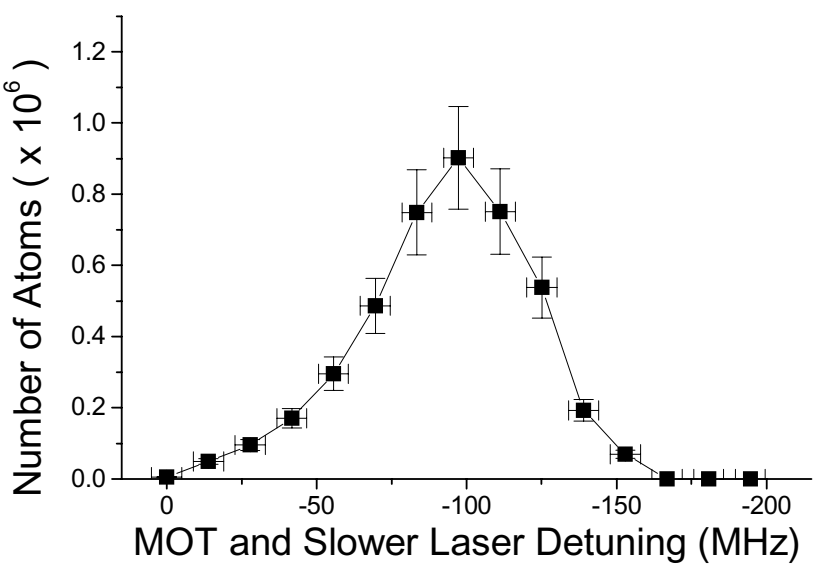

Figure 5. Number of trapped atoms as a function of the laser detuning for a total laser power of 52(1)mW, a vertical field gradient of $63 \mathrm{Gauss} / \mathrm{cm}$ and an oven temperature of $480(1)^{\circ} \mathrm{C}$.

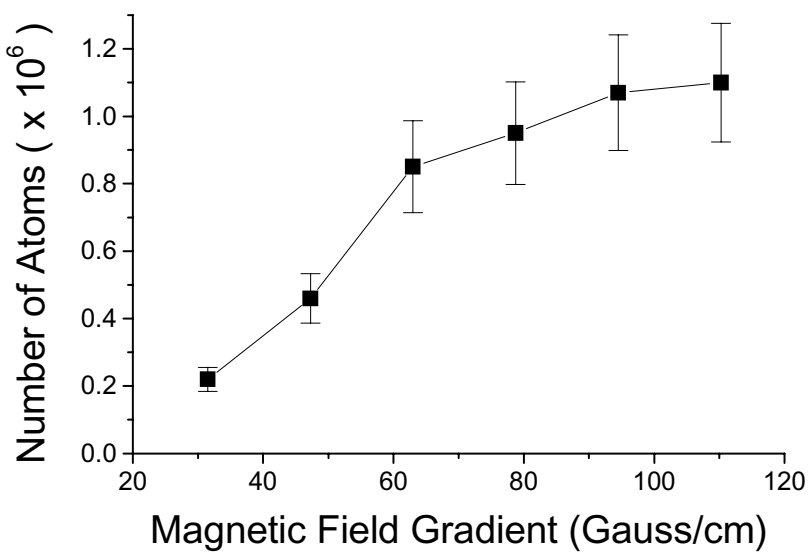

Figure 6. Maximum number of trapped atoms as a function of gradient of the vertical MOT field. Horizontal gradient is half of vertical.

Keeping the power of the trapping beams constant, at 10 $\mathrm{mW}$ per beam, for a vertical field gradient of $63 \mathrm{Gauss} / \mathrm{cm}$ we changed the power of the slower beam and measured the variation in the number of trapped atoms, as shown in Figure 7. The gain in trapped atom number from a slowing power of $2.5 \mathrm{~mW}$ to $5 \mathrm{~mW}$ is more than $100 \%$ while the gain from $5 \mathrm{~mW}$ to $10 \mathrm{~mW}$ is just $30 \%$. This means that it is not necessary to have large laser powers in the Zeeman slowing beam when using low oven temperatures. Of course, larger temperatures will produce larger atom flux that will require more laser power to decelerate the same fraction of the atomic distribution. Figure 8 shows the result of a variation only in the oven temperature, keeping all other parameters fixed (detuning: $-100 \mathrm{MHz}, 10 \mathrm{~mW}$ per each trap and slower beam and a vertical field gradient of $63 \mathrm{Gauss} / \mathrm{cm}$ ).

\section{Decelerating and Trapping With Independent Lasers}

In order to test the efficiency of the focusing slower laser technique we employed an independent laser to decelerate 
the atomic beam. This laser source is a frequency doubled diode laser in an alternative extended cavity, described elsewhere [23]. To measure the doubled diode laser detuning we beat its first harmonic with the Ti:Sapphire laser in a fast photodetector. The thermal drift of the diode laser was determined to be less than $5 \mathrm{MHz}$ per minute, which enable us to perform a series of measurements in a few minutes. The $8.5 \mathrm{~mW} 2^{\text {nd }}$ harmonic output of the diode laser is beam shaped in a telescope to approximately mode match the atomic beam spreading. Keeping the detuning of the MOT beams constant, at -84 (10) $\mathrm{MHz}$, we scan the slower frequency and the result is showed in Figure 9. The maximum number of atoms occurs for a slower detuning of - 438 (15) MHz. By changing the MOT detuning, the optimum value for the slower detuning changes correspondingly, as we can see in Figure 10.

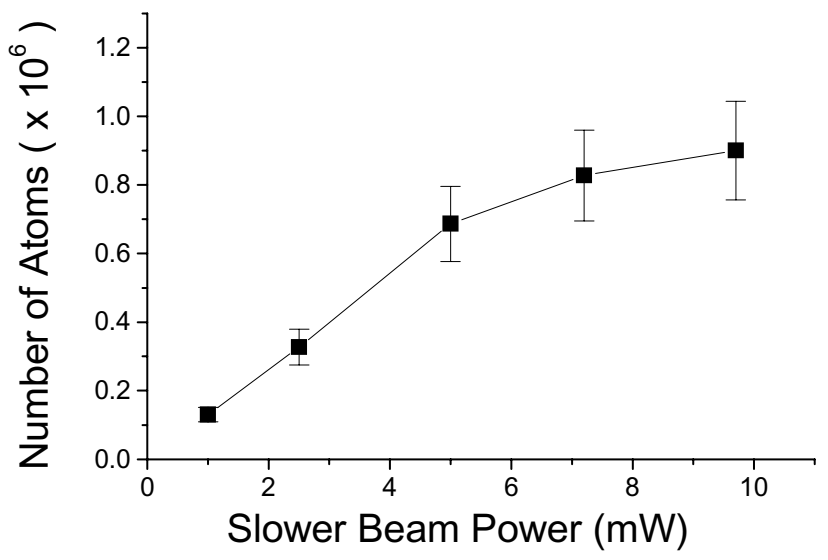

Figure 7. Variation in the power of slower laser beam for the same situation described in Figure 4.

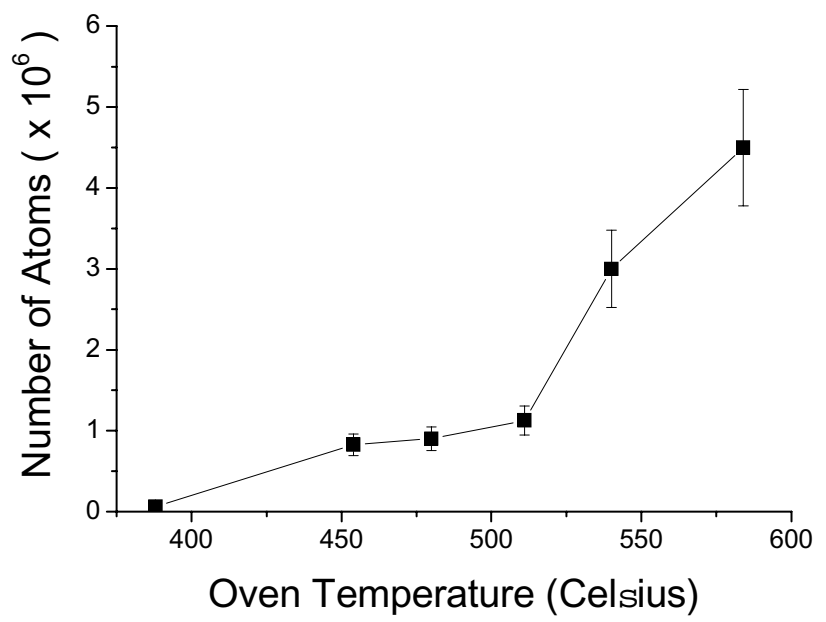

Figure 8. Number of trapped atoms as a function of oven temperature.

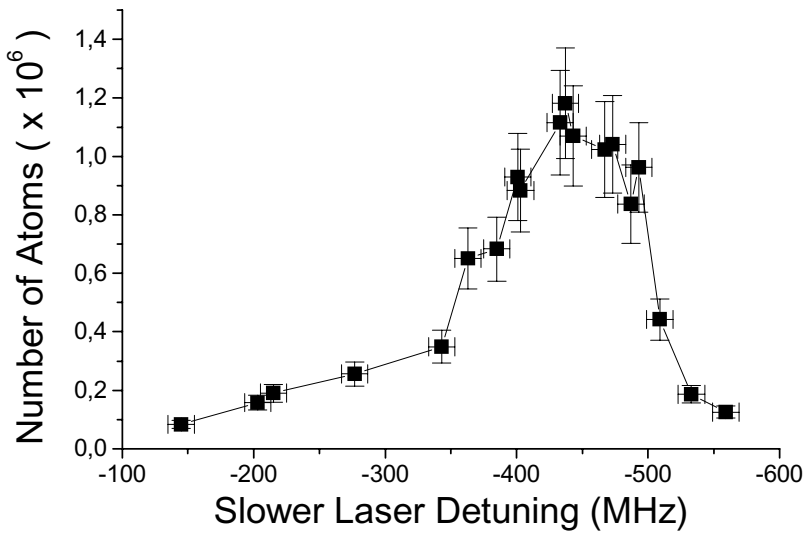

Figure 9. Number of atoms for a fixed MOT detuning of -84 (10) MHz.

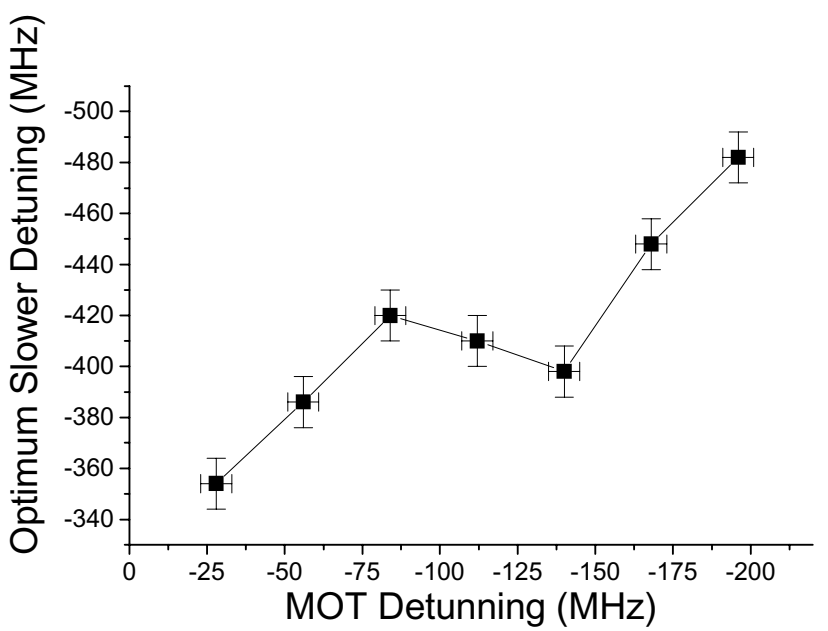

Figure 10. Optimum detuning of the slower laser as a function of detuning of MOT beams (vertical field gradient $=63 \mathrm{Gauss} / \mathrm{cm}$ ).

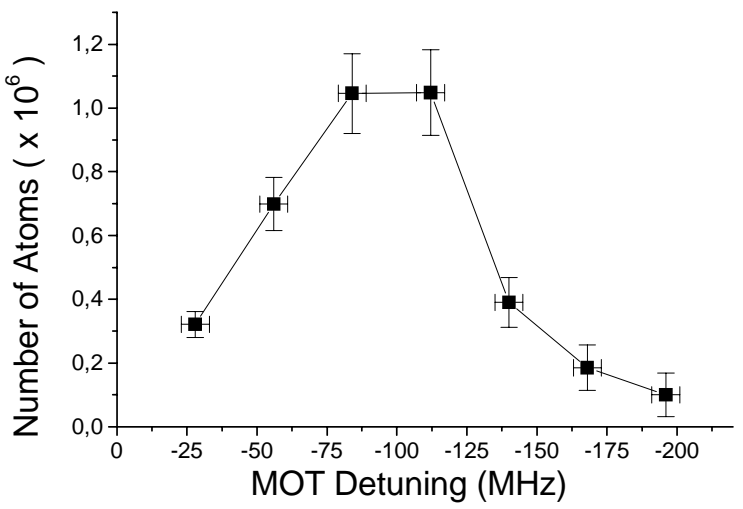

Figure 11. Number of trapped atoms as a function of detuning of the MOT laser, with the slower detuning optimized for each MOT detuning value.

The number of atoms in the situation where the slower is optimized for each value of the MOT detuning is showed in Figure 11, for a trap vertical field gradient of $63 \mathrm{Gauss} / \mathrm{cm}$. We note that the optimum frequency difference between the MOT and the slower beam is practically independent of the MOT detuning. Moreover, the MOT detuning that optimizes the number of atoms is basically the same as when slowing with the same laser. However, with the independent slower 
we could trap $25 \%$ more atoms. This means that the technique of focusing slower has a good efficiency in the trap loading.

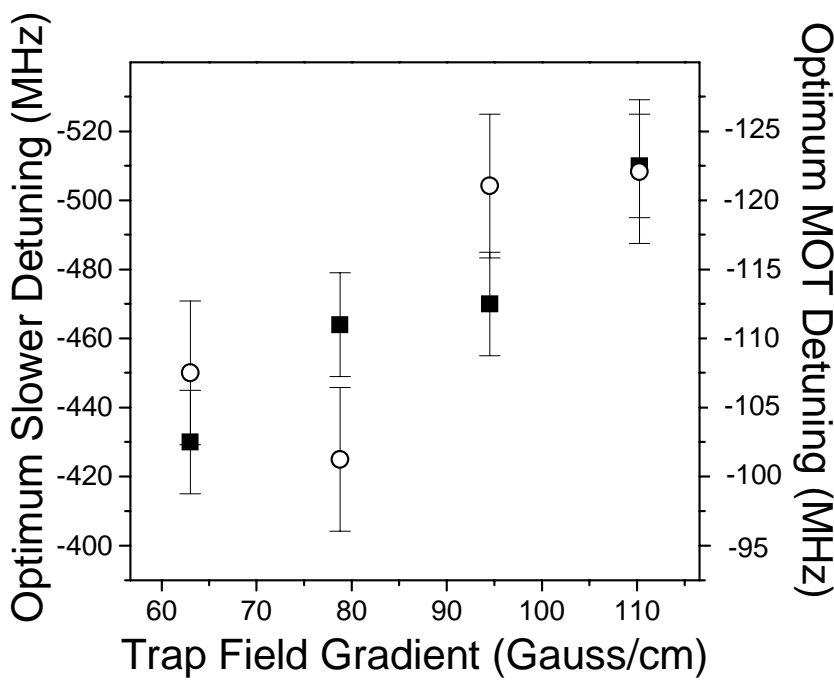

Figure 12. Optimum slower (squares) and MOT (circles) detuning as a function of trap field gradient.

The optimum detuning of the decelerating laser is a compromise between the flux of slow atoms going into the trap and the loss due to the radiation pressure of the slower laser beam in the trapped atoms. This detuning changes considerably with the trap field gradient, although the optimum MOT detuning does not, as we can see in Figure 12.

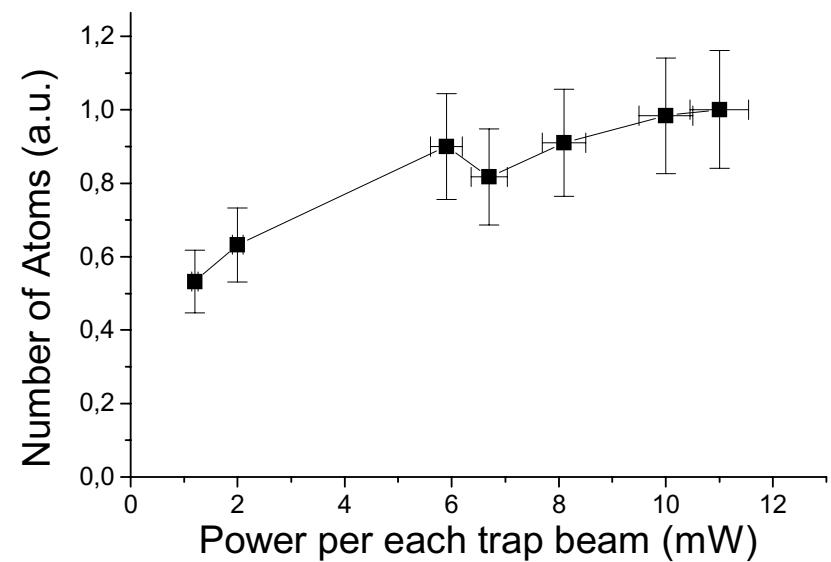

Figure 13. Number of atoms as a function of power on each MOT laser beam.

An interesting result that we could obtain with the independent slower is sketched in Figure 13. It shows the number of trapped atoms when the power in each MOT beam is changed from 1 to $11 \mathrm{~mW}$, while the power and detuning of the slower laser are fixed, causing a constant flux of slow atoms into the MOT capture volume. The result is a very small variation in the number of trapped atoms. A variation of more than ten times in power produces a change of less than two in the number of trapped atoms. Combining this result with that obtained in Figure 7, where we vary the power of the slower beam, we conclude that relatively low power is needed to decelerate a Calcium beam and trap the slowed atoms. Ten milliwatts at $423 \mathrm{~nm}$ is more than suffi- cient, with most of the power into the slower beam to assure adiabatic following during deceleration [16].

We already mentioned that slowing the atomic beam independently increases the number of trapped atoms by $25 \%$. This holds for field gradients below $80 \mathrm{Gauss} / \mathrm{cm}$. By rising the field gradient, the ratio between the optimum number of trapped atoms, when slowing independently or with the focusing laser beam, increases as shown in Figure 14. The maximum field gradient achieved is limited by the heating of our air-cooled anti-Helmholtz coils. For a field gradient of $110 \mathrm{Gauss} / \mathrm{cm}$ and an oven temperature of $580^{\circ} \mathrm{C}$ we obtain a number of $1.2(2) \times 10^{7}$ atoms in the trap.

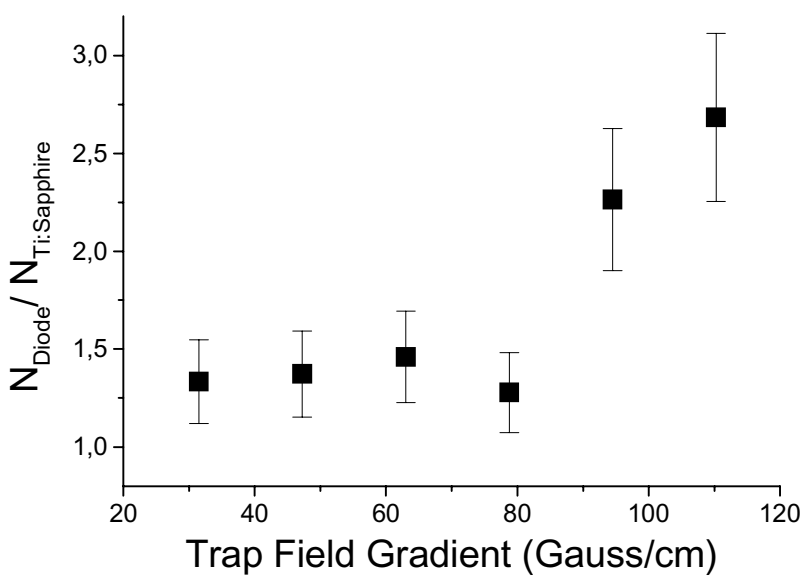

Figure 14. Ratio between the number of trapped atoms when decelerating the atomic bem with an independent laser (diode laser) or with the same laser (Ti:sapphire) used for the MOT.

\section{Storage Time and Temperature}

The modulation of the slower laser beam allows us not only to measure the number of trapped atoms in the background of the atomic beam fluorescence but also the trap lifetime. Figure 15 shows a typical curve of the decay and load process, for low power MOT beams. We observe an exponential decay of 15.4(8) ms, similar to other reported Calcium MOTs [1-4]. The accuracy of this measurement is limited by our detection system. This lifetime is not limited by the imperfect vacuum environment, but by the optical pumping of the ${ }^{1} D_{2}$ level [24]. The transition from the ${ }^{1} P_{1}$ level to the ${ }^{1} D_{2}$ has a probability $10^{5}$ times lower than the probability to decay to the ground state [25]. From the ${ }^{1} D_{2}$ level the atom can decay to the metastable ${ }^{3} P_{2}$ level $(\tau \approx$ 2 hours, [26]), to the ${ }^{3} P_{1}$ or directly to the ground state. By the Einstein coefficients of these transitions, we know that around $22 \%$ of the atoms decay to the ${ }^{3} P_{2}$ level and are lost from the trap. The remaining have some probability to be recaptured, depending on the trap volume [24]. The lifetime of our Calcium MOT shows a behavior similar to that observed for Ytterbium atoms [27]. A detailed analysis of the loss mechanisms in our trap will be described elsewhere [28]. An strategy to increase the lifetime, by decreasing the loss rate and therefore increasing the number of atoms, is to transfer the population of the ${ }^{1} D_{2}$ level to the $5^{1} P_{1}$ level with a repump laser at $672 \mathrm{~nm}$. From the $5{ }^{1} P_{1}$ level the 
atoms decay rapidly to the ground state $\left(1.2 \times 10^{7} \mathrm{~s}^{-1}[25]\right)$ and are recaptured. This scheme was demonstrated by the NIST [3] and the Hamburg group [29], where lifetimes of 84 and $72 \mathrm{~ms}$ were achieved respectively. By lowering the linear loss rate due to the optical pumping of the ${ }^{1} D_{2}$ level, the Hamburg group observed a non-exponential decay, which was attributed to inelastic two-body collisions between cold Calcium atoms. Even without the repump laser at $672 \mathrm{~nm}$, we have observed small differences between the load and decay times, which are also related to cold collisions in the trap [30].

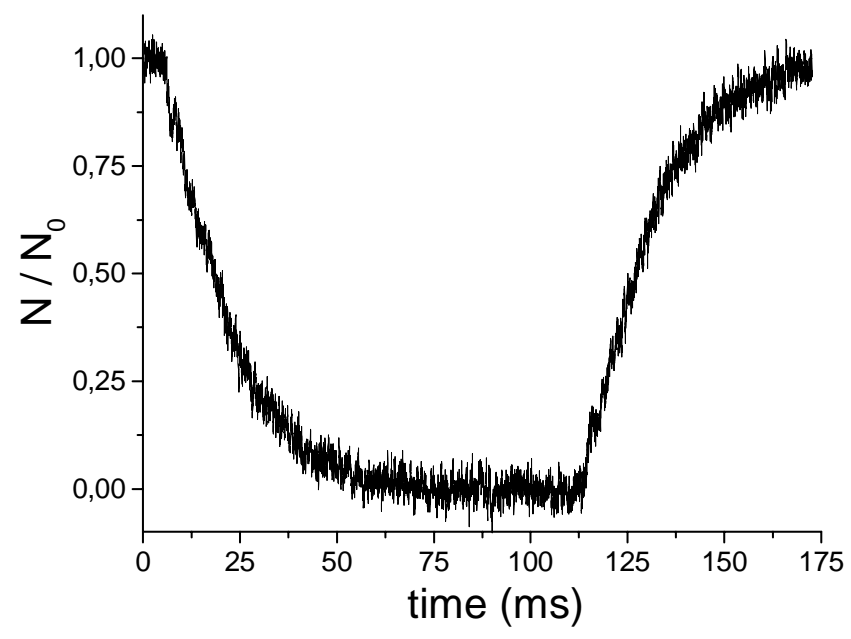

Figure 15. MOT decay and loading curves.

In a MOT, when the Doppler and Zeeman shifts are small compared to the detuning, the radiation pressure force acting on the atoms are in good approximation harmonic [19]. The equipartition of energy implies that the velocity spread and the position spread are related by $m r_{r m s}^{2}=$ $\kappa r_{r m s}^{2}$, where $\kappa$ is the MOT spring constant, given by:

$$
\kappa=8 \hbar \kappa \frac{\delta}{\gamma} \frac{I / I_{s}}{\left(1+I / I_{s}+4 \delta^{2} / \gamma^{2}\right)^{2}}\left(2 \pi \alpha_{B} A\right),
$$

where $I$ is the intensity of the MOT laser beam in the direction considered, $\alpha_{B}$ is the Zeeman splitting of the excited level and $A$ is the magnetic field gradient. Although the number and the lifetime are not critically dependent on the precise MOT alignment, this temperature determination by the cloud size $r_{r m s}$ is. With a careful alignment of the beams we obtain near Gaussian shapes, as those presented in Figure 3. For the parameters of that measurement, we estimate by the horizontal spreading of the atomic cloud a root mean square velocity of $136(12) \mathrm{cm} / \mathrm{s}$, corresponding to a temperature of 9 (2) $\mathrm{mK}$.

\section{Conclusion}

We described in detail our system for laser cooling and magneto-optical trapping of Calcium atoms using the resonant transition ${ }^{1} S_{0}-{ }^{1} P_{1}$ at $423 \mathrm{~nm}$. We have implemented an on-axis magneto-optical trap, loaded from a slowed atomic beam where the deceleration laser has been focused near the trap region. This avoids any perturbation on the trapped atoms due to radiation pressure of the slower laser. We have used a single laser for the MOT and atomic beam slower, which is an important simplification of the apparatus. Decelerating independently the atomic beam with another laser shows that the focusing Zeeman slower technique has efficiency very close to optimum. This also has showed that the number of trapped atoms is practically independent of MOT laser power, in the range from 1 to 11 $\mathrm{mW}$ per trapping beam $\left(16-175 \mathrm{~mW} / \mathrm{cm}^{2}\right)$. We conclude that a total power of $10 \mathrm{~mW}$ at $423 \mathrm{~nm}$ is sufficient to have a Calcium MOT loaded from a decelerated atomic beam. This is particularly important for a practical frequency standard or clock based on cold Calcium atoms.

We derived an expression for the number of atoms when the cloud size is comparable with the trapping laser waist sizes. Several measurements are presented for the dependence of the number of trapped atoms with magnetic field gradient, power and detuning of the trapping lasers, oven temperature, and power and detuning of the decelerating laser. Stable spatial modes of trapped atoms, other than spherical Gaussian, were observed for the first time in a MOT of an alkaline-earth atom. The true $\mathrm{J}^{\prime}=0$ to $\mathrm{J}=1$ cooling transition of these elements allows to perform realistic comparisons between theory and experiment. We have estimated the temperature of the trapped atoms by the size of the atomic cloud. The lifetime of the trap is consistent with the loss mechanism of optical pumping into the metastable ${ }^{1} D_{2}$ level.

\section{Acknowledgements}

We would like to acknowledge the glass shop of the UNICAMP Physics Institute for the very well done work in the construction of the atomic beam and MOT glass chamber. We also would like to thank the machine shop and J.B.Rodrigues for the mechanical parts. This work was supported by FAPESP, CAPES and CNPq and FAEPUNICAMP, Brazilian government agencies. Support for RLCF was through CAPES graduate research scholarship and for DAM and DRO through FAPESP graduate and undergraduate scholarships respectively.

\section{References}

[1] Th. Kisters, K. Zeiske, F. Riehle and J. Helmcke, Appl. Phys. B 59, 89 (1994).

[2] The first demonstration of magneto-optical trapping of alkaline-earth elements was reported in: T. Kurosu and F. Shimizu, Jpn. J. Appl. Phys. 29, L2127 (1990).

[3] C. W. Oates, F. Bondu, R. W. Fox and L. Holberg, Eur. Phys. J. D 7, 449 (1999).

[4] J. Grünert, G. Quehl, V. Elman and A. Hemmerich, J. Mod. Opt. 47, 2733 (2000).

[5] T. Kurosu, G. Zinner, T. Trebst and F. Riehle, Phys. Rev. A 58, R4275 (1998).

[6] F. Riehle, H. Schnatz, G. Zinner, K. Zeiske, B. Lipphardt and J. Helmcke, Laser Phys. 6, 237 (1996). 
[7] P. Kersten, F. Mensing, U. Sterr and F. Riehle, Appl. Phys. B 68, 27 (1999).

[8] T. Binnewies, G. Wilpers, U. Sterr, F. Riehle, J. Helmcke, T. E. Mehlstaubler, E. M. Rasel and W. Ertmer, Phys. Rev. Lett 87, 3002 (2001).

[9] E. A. Cutis, C. W. Oates and L. Hollberg, Phys. Rev. A 64, 1403 (2001).

[10] T. Udem, S.A. Diddams, K. R. Vogel, C. W. Oates, E. A. Curtis, W. D. Lee, W. M. Itano, R. E. Drullinger, J. C. Bergquist and L. Hollberg , Phys. Rev. Lett. 86, 4996 (2001).

[11] J. V. B. Gomide, G. A. Garcia, F. C. Cruz, A. J. Polaquini, M. P. Arruda, D. Pereira and A. Scalabrin, Brazilian J. Phys. 27, 266 (1997); G. Woehl, G.D. Garcia, F.C. Cruz, D. Pereira and A. Scalabrin, Appl. Opt. 38, 2540 (1999).

[12] An efficient Calcium atomic beam system has been designed by the Hamburg group [5], where practically all the atoms coming out of the oven contribute to the atomic beam. This system allowed them to trap the record number of $10^{8} \mathrm{Cal}-$ cium atoms, for an oven temperature of $650^{\circ} \mathrm{C}$.

[13] J. A. Neuman, P. Wang and A. Gallagher, Rev. Sci. Instrum. 66, 3021 (1995).

[14] H. J. Onisto, R.L. Cavasso Filho, A. Scalabrin, D. Pereira and F.C. Cruz, ; Opt. Engineering 41 5, 1122 (2002).

[15] E. L. Raab, M. Prentiss, A. Cable, S. Chu and D.E. Pritchard, Phys. Rev. Lett. 59, 2631 (1987).

[16] R. J. Napolitano, S. C. Zlio and V. S. Bagnato, Opt. Commun. 80, 110 (1990).

[17] S. G. Miranda, S. R. Muniz, G. D. Telles, L. G. Marcassa, K. Helmerson and V. S. Bagnato, Phys. Rev. A 59, 882 (1999).
[18] P. D. Lett, W. D. Phillips, S. L. Rolston, C. E. Tanner, R. N. Watts and C. I. Westbrook, J. Opt. Soc. Am. B 6, 2084 (1999).

[19] H. J. Metcalf and P. van der Straten, Laser cooling and trapping (Springer-Verlag NY, Inc., 1999).

[20] T. P. Dinneen, K. R. Vogel, E. Arimondo, J. L. Hall and A. Gallagher, Phys. Rev. A 59, 1216 (1999).

[21] I. Guedes, M. T. Dearaujo, D. M. B. P. Milori, G. I. Surdutovich, V. S. Bagnato and S. C. Zilio, J. Opt. Soc. Am. B 11, 1935 (1994).

[22] D. Felinto and S. S. Vianna, J. Opt. Soc. Am. B 17, 681 (2000).

[23] D. A. Manoel, R. L. Cavasso Filho, A. Scalabrin, D. Pereira and F. C. Cruz, Opt. Commun. 201, 157 (2002).

[24] T. Kurosu and F. Shimizu, Jpn. J. Appl. Phys. 31, 908 (1992).

[25] N. Beverini, F. Giammanco, E. Maccioni, F. Strumia and G. Vissani, J. Opt. Soc. Am. B 6, 2188 (1989).

[26] A. Derevianko, Phys. Rev. Lett. 87, 3002 (2001).

[27] T. Loftus, J. R. Bochinski, R. Shivitz and T. W. Mossberg, Phys. Rev. A 61, R51401 (2000).

[28] R. L. Cavasso Filho, A. Scalabrin, D. Pereira and F. C. Cruz, Excited-state-population-dependent loss from a Calcium magneto-optical trap, Europhys. J. D (submitted, 2002).

[29] J. Grünert and A. Hemmerich, Appl. Phys. B 73, 815 (2001).

[30] R. L. Cavasso Filho, A. Scalabrin, D. Pereira and F.C. Cruz, Observing negligible collision trap losses: the case of alkaline -earth elements, Phys. Rev. A67, 0214022003. 